\title{
THE POISSON KERNEL FOR DRINFELD MODULAR CURVES
}

\author{
JEREMY T. TEITELBAUM
}

\section{INTRODUCTION}

In an earlier work [ $\mathrm{T} 1$ ], the author described a technique for constructing rigid analytic modular forms on the $p$-adic upper half plane by means of an integral transform (a "Poisson Kernel"). In this paper, we apply these methods to the study of rigid analytic modular forms on the upper half plane over a complete local field $\hat{k}$ of characteristic $p$. Arising out of the theory of Drinfeld modules [D], these modular forms, first studied by Goss (see [Go1, Go2]), are in many ways analogous to the classical modular forms for $\mathrm{SL}_{2}(\mathbb{Z})$. For a brief, but effective, introduction to the theory of these "characteristic $p$ " modular forms, see the introduction to Gekeler's paper [Gek1]. Also useful is Gekeler's book [Gek2].

We establish three main results. First of all, we show that if, $k$ is the function field of a complete, geometrically irreducible curve $C$ over the finite field $\mathbb{F}_{q}$ with $q$ elements, $\hat{k}$ is a completion of $k, \Gamma$ is an arithmetic subgroup of $\mathrm{GL}_{2}(k)$, and $\mu$ is a measure on $\mathbb{P}_{\hat{k}}^{1}$ coming from a "harmonic cocycle of weight $n$ for $\Gamma$," (see Definition 1 below) then

$$
F(z)=\int_{\mathbb{P}_{\hat{k}}^{1}} \frac{1}{z-x} d \mu(x)
$$

is a cusp form of weight $n$ for $\Gamma$. This generalizes Theorem 3 of [T1] to the characteristic $p$ case.

Next we show that every cusp form for an arithmetic subgroup $\Gamma$ of $\mathrm{GL}_{2}(k)$ can be represented by an integral like $(*)$. This result can be interpreted as an isomorphism between certain relative homology groups of $\Gamma$ and corresponding spaces of cusp forms (see Proposition 21). As in the characteristic zero case covered in [T1], this result is a consequence of the Riemann-Roch theorem and analysis on the tree associated to $\mathrm{GL}_{2}(k)$. The calculations of the dimensions of spaces of modular forms carried out by Goss and Gekeler also play an important role. The characteristic $p$ result is more technical than the result in [T1], due

Received by the editors June 20,1990; presented at the workshop on the Arithmetic of Function Fields, Ohio State University, June 1991.

1991 Mathematics Subject Classification. Primary 11R58, 11F67, 11 F85.

This research was partially supported by an NSF Postdoctoral Fellowship. 
to the presence of cusps. Our proof relies on the theory of the Euler-Poincare characteristic discussed in [Se, Chapter II].

Finally, we apply the integral representation $(*)$ to give an integral formula for the coefficients in the Taylor expansion of a cusp form for a congruence subgroup of $\mathrm{GL}_{2}\left(\mathbb{F}_{q}[T]\right)$ at a cusp. For example, suppose that $f$ is a form for $\mathrm{SL}_{2}\left(\mathbb{F}_{q}[T]\right)$, that $e$ and $\bar{\pi}$ are respectively the exponential function for the Carlitz module and its period, and that $t(x)=1 / e(\bar{\pi} x)$. Then $f$ has a Fourier-type expansion $f=\sum_{i \geq 1} b(i) t^{i}$ at infinity and we show that

$$
b(i)=\bar{\pi} \int_{\mathbb{F}_{q}\left(\left(\frac{1}{T}\right)\right) / \mathbb{F}_{q}[T]} e(\bar{\pi} x)^{i-1} d \mu_{f},
$$

where $\mu_{f}$ is the measure giving $f$. The formula is similar for a congruence subgroup. These coefficients have been computed by a variety of techniques (see the works of Goss and Gekeler). Our formula should be compared to the classical Fourier transform formula for the Fourier coefficients of a modular form for $\mathrm{SL}_{2}(\mathbb{Z})$. Perhaps it will shed some light on the relationship between modular forms for $\mathrm{GL}_{2}(k)$ and Goss' $L$-functions [Go3].

The author thanks D. Goss and and E. U. Gekeler for their helpful comments on this work.

\section{Preliminaries}

Notation. Let $k$ be the field of functions on a complete, geometrically irreducible curve $C$ over the finite field $\mathbb{F}_{q}$ with $q$-elements. Fix a place $m$ of $k$ of degree $\delta$, and let $\hat{k}$ be the completion of $k$ at this place. Let $K$ be a completion of an algebraic closure of $\hat{k}$. Let $T^{-1}$ be a uniformizing parameter for $\hat{k}$; let $\operatorname{deg}$ be a valuation at $m$ with $\operatorname{deg} T=\delta$, and let $|\cdot|$ be the associated absolute value extended to $K$. Finally, let $A$ be the subring of $k$ of functions with poles only at $m$.

Let $\mathscr{T}$ be the tree of $\mathrm{GL}_{2}(\hat{k})$ and $\Omega=K-\hat{k}$ be Drinfeld's "upper half plane." The group $\mathrm{GL}_{2}(\hat{k})$ acts on $\Omega$ by linear fractional transformations in $z$. There is a reduction map red: $\Omega \rightarrow \mathscr{T}$ that commutes with the action of $\mathrm{GL}_{2}(\hat{k})$ on $\mathscr{T}$.

In order to clarify the relationship between the tree $\mathscr{T}$ and the upper half plane $\Omega$, we will make the reduction map a bit more explicit. For more details on this construction, the reader may refer to [D], [GvdP], or [T2].

Given a rational number $r$ and an element $a$ of $k$, let

$$
B(a, r)=\left\{z \in K:|z-a| \leq q^{r \delta}\right\} \text {. }
$$

Now let $U(1)$ be the admissible open subset of $\Omega$ defined by

$$
U(1)=\left\{z \in K: q^{-\delta}<|z|<q^{\delta}\right\}-\bigcup B(a,-1),
$$

where the union is over all $a \in \mathbf{F}_{q^{\delta}}^{*}$. The set $U(1)$ is an "open annulus" punctured at the rational points. 
It is not hard to check that translates of $U(1)$ by elements of $\mathrm{GL}_{2}(k)$ cover $\Omega$. In fact, if we set $U(\gamma)=\gamma^{-1} U(1)$, we find that $U(\gamma)=U\left(\gamma^{\prime}\right)$ precisely when $\gamma=\tau \gamma^{\prime}$ for $\tau \in \mathrm{GL}_{2}(\mathscr{O})$, where $\mathscr{O}$ is the valuation ring in $\hat{k}$. This gives an identification between $\mathrm{GL}_{2}(\mathscr{O}) \backslash \mathrm{GL}_{2}(k)$ and the distinct $U(\gamma)$. This coset space is precisely the set of vertices of the tree $\mathscr{T}$.

One may recover the entire tree $\mathscr{T}$ as the nerve of the covering of $\Omega$ by the $U(\gamma)$. Indeed, two different $U(\gamma)$ will overlap on an annulus, which is $\mathrm{GL}_{2}(k)$ equivalent to the basic annulus

$$
V=\left\{z: 1<|z|<q^{\delta}\right\} .
$$

Having realized $\mathscr{T}$ as the nerve of the covering of $\Omega$ by the $U(\gamma)$, we may construct the reduction map "by hand." We decree first of all that, if $z$ is a point in $\Omega$ that belongs to precisely one of the $U(\gamma)$, then $z$ reduces to the corresponding vertex of $\mathscr{T}$. If $z$ belongs to two distinct $U(\gamma)$, then $z$ reduces to a point on the corresponding edge. Such an edge corresponds to an annulus in $\Omega$; to make the correspondence between points on such an annulus and points on the associated edge of $\mathscr{T}$, we need the idea is of an oriented annulus.

Definition 1. An orientation on an annulus $W$ is represented by an isomorphism

$$
w: W \rightarrow V .
$$

Two such isomorphisms $w$ and $w^{\prime}$ represent the same orientation if the function $u=\left(w^{-1} \circ w^{\prime}\right)^{*} z$ on $V$ satisfies $|u(P)|=|z(P)|$ at all points $P$ of $V$.

One can show that a given annulus has precisely two orientations; for example $V$ has its canonical orientation (determined by the identity map, or, equivalently, the function $z$ ) and its opposite orientation (determined by the function $T / z$.)

We associate to the annulus $V$, with its canonical orientation, the oriented edge $e=[U(1), U(\tau)]$, where

$$
\tau=\left(\begin{array}{ll}
0 & T \\
1 & 0
\end{array}\right) .
$$

In terms of a barycentric coordinate $x$ on $e$, a point $z$ reduces to the point on $e$ where $x=\delta^{-1} \log _{q}|z|$. If we extend this map to all of $\Omega$ by $\mathrm{GL}_{2}(k)$ equivariance, we obtain the entire reduction map.

The space of ends $E$ of the tree $\mathscr{T}$ plays a crucial role in this paper. This space consists of the set of infinite rays in $\mathscr{T}$, modulo the relation that identifies two rays if they differ by a finite initial segment. Each oriented edge $e$ of $\mathscr{T}$ defines an open subset $U(e)$ of $E . U(e)$ consists of the set of rays that pass through $e$. As is well known, $E$, with this topology, is homeomorphic to $\mathbb{P}_{\hat{k}}^{1}$. Let $\overline{\mathscr{T}}$ denote the compactification of $\mathscr{T}$ obtained by adjoining the ends. The reduction map red $: \Omega \rightarrow \mathscr{T}$ extends to a map $\mathbb{P}_{\hat{k}}^{1} \rightarrow \overline{\mathscr{T}}$, which respects the $\mathrm{GL}_{2}(\hat{k})$ action and induces a homeomorphism of $\mathbb{P}_{\hat{k}}^{1}$ with $E$. Therefore we 
may find a homeomorphism $x: E \rightarrow \mathbb{P}_{\hat{k}}^{1}$ such that

$$
x(\gamma u)=\frac{a x(u)+b}{c x(u)+d} .
$$

We will tend to abuse notation and identify $E$ with $\mathbb{P}_{\hat{k}}^{1}$ by means of the function $x$.

Let us also establish some terminology derived from that of [T1].

Definition 2. Let $M$ be an abelian group and $\Gamma$ a subgroup of $\mathrm{GL}_{2}(k)$.

(1) An $M$-valued function $c$ on the edges of $\mathscr{T}$ is called a harmonic cocycle if

(a) For all vertices $v$ of $\mathscr{T}$,

$$
\sum_{e \mapsto v} c(e)=0
$$

where the sum is over the oriented edges $e$ of $\mathscr{T}$ meeting $v$.

(b) If $e^{*}$ denotes $e$ with the orientation reversed, then $c\left(e^{*}\right)=-c(e)$ for all edges $e$ of $\mathscr{T}$.

(2) For integers $n \geq 0$, let $V(n)$ be the space of homogeneous forms in two variables $X$ and $Y$ of degree $n-1$ over the field $\hat{k}$. If

$$
\gamma=\left(\begin{array}{ll}
a & b \\
c & d
\end{array}\right) \in \mathrm{GL}_{2}(\hat{k})
$$

then let $\gamma$ act on $V(n)$ by

$$
\gamma \cdot\left(\begin{array}{l}
X \\
Y
\end{array}\right)=\frac{1}{(a d-b c)}\left(\begin{array}{cc}
d & -b \\
-c & a
\end{array}\right)\left(\begin{array}{l}
X \\
Y
\end{array}\right) .
$$

By convention, let $V(-n)=\operatorname{Hom}(V(n), \hat{k})$. Then we define $C_{\mathrm{har}}(\Gamma, n)$ to be the space of $\Gamma$-invariant harmonic cocycles taking values in the space $V(1-n) \otimes(\mathrm{det})^{-1}$. Such a harmonic cocycle satisfies the condition

$$
c(\gamma e)\left(X^{i} Y^{n-2-i}\right)=(a d-b c) c(e)\left((a X+b Y)^{i}(c X+d Y)^{(n-2-i)}\right) \text {. }
$$

Elements of $C_{\text {har }}(\Gamma, n)$ will be called harmonic cocycles of weight $n$ (for $\Gamma$ ).

A subgroup $\Gamma$ of $\mathrm{GL}_{2}(k)$ is called arithmetic if there is a rank two, projective $A$ submodule $Y$ of $k \oplus k$ such that $\Gamma$ is contained in $\operatorname{GL}(Y)$ and contains the kernel $\mathrm{GL}(Y, I)$ of the map $\mathrm{GL}(Y) \rightarrow \mathrm{GL}(Y / I Y)$ for some ideal $I \subset A$. An important example of such subgroups are the congruence subgroups $\Gamma(I)$, defined as the kernel of the natural map from $\mathrm{GL}_{2}(A) \rightarrow \mathrm{GL}_{2}(A / I)$. (See [Gek2, V.2].)

\section{CUSPIDAL HARMONIC COCYCLES AND MEASURES}

Cuspidal cocycles. As we have remarked earlier, the most significant difference between the theory we develop in this paper for $\mathrm{GL}_{2}(k)$ and our earlier work on $\mathrm{SL}_{2}\left(\mathbb{Q}_{p}\right)$ is the presence of cusps for $\mathrm{GL}_{2}(k)$ on $\Omega$. In this section, we examine the behavior of harmonic cocycles near cusps, and show that every harmonic cocycle vanishes "near a cusp." 
Definition 2. A cusp of an arithmetic subgroup $\Gamma$ is an end of the quotient tree $\mathscr{T} / \Gamma$.

The quotient $\mathscr{T} / \Gamma$ is a finite graph with a finite number of cusps attached (See [Se,Chapter II, Theorem 9].) In the theory of complex-valued automorphic forms for $\Gamma$ (see [W]), one is concerned with cuspidal, complex-valued harmonic cocycles-that is, harmonic cocycles that vanish off of a finite subgraph of $\mathscr{T} / \Gamma$. As we see in the next theorem, this condition is automatically satisfied when one considers cocycles taking values in characteristic $p$.

Theorem 3. Suppose $\Gamma \subset \mathrm{GL}_{2}(k)$ is an arithmetic subgroup and $M$ is a finitedimensional $\hat{k}$ vector space on which $\Gamma$ acts. Then every $\Gamma$-equivariant, $M$ valued harmonic cocycle is cuspidal-that is, it vanishes off of a finite set of $\Gamma$-equivalence classes of edges of $\mathscr{T}$.

Proof. Let $p$ be a cusp for $\Gamma$, and let $e_{1}, e_{2}, \ldots, e_{k}, \ldots$ be a sequence of edges in $\mathscr{T}$ representing $p$. We will show that $c$ vanishes on all $e_{i}$ for $i$ sufficiently large. This implies that $c$ is nonzero on only finitely many edges in $\mathscr{T} / \Gamma$. We will assume, without loss of generality, that $p$ is the cusp at infinity.

Let $U$ be the maximal unipotent subgroup of $\Gamma$ that fixes $p$. $U$ contains a maximal subgroup consisting of matrices of the form

$$
\left(\begin{array}{ll}
1 & x \\
0 & 1
\end{array}\right)
$$

where $x$ belongs to a fractional ideal $L$ of $A$. Define a sequence of subgroups

$$
U_{i}=\left\{\gamma \in U: \gamma e_{i}=e_{i}\right\}
$$

We have

$$
U_{1} \subset U_{2} \subset \cdots \subset U_{n} \subset \cdots
$$

and

$$
\bigcup U_{i}=U
$$

Since $\Gamma$ contains a conjugate of the group of translations $z \mapsto z+b$, we may drop an initial finite set of edges from our representative for $p$, and assume that the sequence of $U_{i}$ is strictly increasing. In this case, $U_{i} / U_{i-1}$ is cyclic of order $q^{\delta}$.

Suppose $c$ is a harmonic cocycle for $\Gamma$ taking values in $M$, which is finite dimensional over $\hat{k}$. Let us define $M_{i}$ to be the $U_{i}$ invariant elements in $M$. Clearly $M_{i+1} \subset M_{i}$ for all $i$. The $\Gamma$-equivariance of $c$ implies that $c\left(e_{i}\right) \in M_{i}$.

Since the $M_{i}$ are a descending sequence of subspaces in the finite-dimensional space $M$, there is an $N$ such that, for all $i \geq N$, we have $M_{i}=M_{i+1}$. Choose $j>N$. The group $U_{j}$ fixes $e_{j}$ and therefore the vertex $v_{j}$, which joins $e_{j-1}$ to $e_{j}$. The group $U_{j-1}$ also fixes $v_{j}$, and $U_{j} / U_{j-1}$ transitively permutes the $q^{\delta}$ edges other than $e_{j-1}$, which leave $v_{j}$. Let $\left\{\gamma_{1}, \ldots, \gamma_{q^{\delta}}\right\}$ represent the elements of $U_{j} / U_{j-1}$. Recalling that $c$ is harmonic and that 
$c\left(e_{j-1}\right) \in M_{j-1}=M_{j}$, we obtain

$$
\begin{aligned}
c\left(e_{j}\right) & =-\sum_{i=1}^{q^{\delta}} c\left(\gamma_{i} e_{j-1}\right)=-\sum_{i=1}^{q^{\delta}} c\left(e_{j-1}\right) \\
& =-q^{\delta} c\left(e_{j-1}\right)=0 .
\end{aligned}
$$

Therefore, $c$ vanishes on all $e_{j}$ for $j>N$, and, consequently, $c$ is cuspidal.

Measures. Generalizing earlier work of Drinfeld and Schneider, we showed in [T1] how to construct measures on $\mathbb{P}^{1}\left(\mathbb{Q}_{p}\right)$ from harmonic cocycles on the tree of $\mathrm{SL}_{2}\left(\mathbb{Q}_{p}\right)$. Here we generalize these results to $\mathrm{GL}_{2}(k)$, with most of the work devoted to understanding the effect of the cusps.

For $n \geq 2$, let $\mathscr{A}_{n}$ denote the set of $\hat{k}$-valued functions on $\mathbb{P}_{\hat{k}}^{1}$ (viewed as the ends of $\mathscr{T}$ ), which are locally meromorphic, with poles only at $\infty$ of order at most $n-2$. We point out that $\mathscr{A}_{n}$ contains the space $P_{n}$ of polynomials of degree $n-2$ in $x$.

Definition 4. Let $c \in C_{\text {har }}(\Gamma, n)$ be a harmonic cocycle of weight $n \geq 2$. Given an edge $e$ of $\mathscr{T}$ and an integer $0 \leq i \leq n-2$, we define

$$
\int_{U(e)} x^{i} d \mu_{c}(x)=c(e)\left(X^{i} Y^{n-2-i}\right),
$$

and extend this definition to $P_{n}$ by linearity.

The properties of $c$ have the following two consequences.

Lemma 5. The integral defined by $c \in C_{\mathrm{har}}(\Gamma, n)$ on $P_{n}$ has the following properties:

(1) $\int_{U(\gamma e)} f(x) d \mu=\int_{U(e)} \operatorname{det}(\gamma) f(\gamma x)(c x+d)^{n-2} d \mu$.

(2) $\int_{\mathbb{P}_{\grave{k}}^{1}} f(x) d \mu=0$.

Proof. The first of these two properties follows from the transformation law for $c \in C_{\text {har }}(\Gamma, n)$ and the second follows from the fact that $c$ is harmonic.

The integral we have constructed on $P_{n}$ extends to $\mathscr{A}_{n}$. To carry out the extension, we require only the following estimate.

Lemma 6. There exists a constant $C>0$ such that, for all $0 \leq i \leq n-2$,

$$
\left|\int_{U(e)}(x-r)^{i} d \mu\right|<C \rho(e)^{i-(n-2) / 2}
$$

where $e$ is an edge of $\mathscr{T}$ with $\infty \notin U(e), r \in U(e)$, and $\rho(e)=$ $\sup _{x, y \in U(e)}|x-y|$ is the diameter of $U(e)$.

Proof. The proof of this lemma is obtained directly from [Sch, pp. 227-228] by making some slight changes in notation. We include it only for the sake of 
completeness. First one shows by a calculation that, for all $r \in \hat{k}$ such that $\gamma r \neq \infty$ and $0 \leq i \leq n-2$, we have

(1) $\int_{U(e)}(x-\gamma r)^{i} d \mu=(c r+d)^{n-2 i} \sum_{j=0}^{n-i}\left(\begin{array}{c}n-i \\ j\end{array}\right)\left(r+\frac{d}{c}\right)^{-j} \int_{U(e)}(x-r)^{i+j} d \mu$.

If $e$ is $\Gamma$-equivalent to an edge $e^{\prime}$ such that $c\left(e^{\prime}\right)=0$, then our desired estimate is obtained automatically. Suppose, therefore, that $e_{1}, \ldots, e_{m}$ are a set of representatives for the edges in $\mathscr{T} / \Gamma$ where $c$ is nonzero. (Recall that, by Theorem 3, this set is indeed finite.) Suppose that $e=\gamma e_{s}$, that $\rho_{s}$ is the diameter of $U\left(e_{s}\right)$, and that $r \in U(e)$. Then

$$
\left|\gamma^{-1}(r)+\frac{d}{c}\right|=\left|\gamma^{-1}(r)-\gamma^{-1}(\infty)\right| \geq \rho_{s}
$$

and

$$
\rho(e)=\left|c \gamma^{-1}(r)+d\right|^{-2} \rho_{s} .
$$

From these estimates and equation (1) we obtain

$$
\rho(e)^{(n-2) / 2-i}\left|\int_{U(e)}(x-e)^{i} d \mu\right| \leq \max _{0 \leq j \leq n-2-i} \rho_{s}\left|\int_{U\left(e_{s}\right)}\left(x-\gamma^{-1} r\right)^{i+j} d \mu\right|
$$

and the second term in this inequality can be bounded absolutely, independent of $\gamma^{-1} r \in U(e)$.

Just as in the characteristic zero case described in [T1], this estimate allows us to extend our integral from $P_{n}$ to the larger class of functions $\mathscr{A}_{n}$. For the sake of reference we reproduce the properties of the extension here.

Proposition 7. Let $c \in C_{\mathrm{har}}(\Gamma, n)$, and let $\mu$ be the associated measure on $\mathscr{A}_{n}$. Then

(1) $\int_{U} f d \mu$ is finitely additive in $U$ and linear in $f$ for $f \in \mathscr{A}_{n}$ and $U \subset \mathbb{P}_{\hat{k}}^{1}$.

(2) For $0 \leq i \leq n-2$,

$$
\int_{U(e)} x^{i} d \mu=c(e)\left(X^{i} Y^{n-2-i}\right) .
$$

(3) There exists a constant $C$ such that if $\infty \in U(e), 0 \notin U(e)$, and $-\infty \leq i \leq n-2$, we have

$$
\int_{U(e)} x^{i} d \mu \leq C \rho(e)^{-i+(n-2) / 2}
$$

while if $a \in U(e) \subset \hat{k}$ and $i \geq 0$, then

$$
\int_{U(e)}(x-a)^{i} d \mu \leq C \rho(e)^{i-(n-2) / 2} .
$$


(4) Suppose $\infty \in U(e)$ and that $F(x)=\sum_{i=n-2}^{-\infty} a_{n} x^{n}$ is a Laurent expansion for $F$ that converges on the set $U(e)-\{\infty\}$. Then

$$
\int_{U(e)} F d \mu=\sum a_{i} \int_{U(e)} x^{i} d \mu .
$$

If $\infty \notin U(e), \lambda$ is a center for $U(e)$, and $F(x)=\sum_{i=0}^{\infty} a_{i}(x-\lambda)^{i}$ is a convergent Taylor series for $F$ on $U(e)$, then

$$
\int_{U(e)} F d \mu=\sum a_{i} \int_{U(e)}(x-\lambda)^{i} d \mu .
$$

Let us briefly explain how the integral whose existence is asserted in this proposition is computed. Essentially, one approximates $f$ locally by polynomials of degree $n-2$, constructs Riemann sums, and passes to a limit. Suppose that $e$ is an edge of $\mathscr{T}$, and $U(e)$ is the corresponding compact open in $k$. Let $f$ be a locally analytic function on $U(e)$. Then $f$ can be expanded in a Taylor series on $U(e)$; we let $P(f, e)$ be the polynomial obtained from this series by dropping the terms of degree greater than $n-2$. (One can show that the choice of center point in $U(e)$ to make the Taylor expansion does not matter.) Then by Proposition 7(2), we know how to compute the integral of the polynomial $P(f, e)$ on $U(e)$. To compute the integral of $f$, we set

$$
\int_{U(e)} f(x) d \mu(x)=\lim \sum_{U\left(e^{\prime}\right)} \int P\left(f, e^{\prime}\right) d \mu(x),
$$

where the limit is taken over partitions of $U(e)$ into smaller compact opens $U\left(e^{\prime}\right)$. The properties of the integral guarantee that these generalized Riemann sums converge.

If infinity belongs to $U(e)$, one follows the same procedure, except that one expands $f$ into a Laurent series in $1 / x$, then truncates the terms involving negative powers of $x$. The resulting truncation is a polynomial of degree at most $n-2$, since $f$ has at worst a pole of that order at infinity.

This concludes our discussion of measures derived from cocycles. In the next section, we will show how to use these measures to construct cusp forms.

\section{The $T$-ADic Poisson Kernel}

Modular forms. In this section, we actually construct modular forms from harmonic cocycles. First, we must define the modular forms we are studying, and to do that we need to introduce two functions from the theory of Drinfeld modules.

Let $L$ be a nonzero fractional ideal of $A$. Define

$$
e_{L}(z)=z \prod_{\substack{a \in L \\ a \neq 0}}(1-z / a) \text { and } t_{L}(z)=e_{L}^{-1} .
$$

As explained, for example, in [Gek2, Chapter I, §2], these functions are rigid analytic on $\Omega, \mathbb{F}_{q}$-linear, and invariant under $L$-translations. The functions 
$t_{L}$ serve as uniformizing parameters "at infinity" for $\Omega$, the role played by $\exp (2 \pi i n z)$ in the classical theory of modular forms.

Suppose that $p$ is a cusp for $\Gamma$ and that $\mu \in \mathrm{GL}_{2}(k)$ satisfies $\mu(\infty)=p$. If $\Gamma(p)$ denotes the stabilizer of $p$, then $\mu^{-1} \Gamma(p) \mu$ fixes $\infty$, and therefore contains a maximal subgroup consisting of translations $z \mapsto z+b$ for $b$ in some fractional ideal $L$ of $A$. Define $t(p, \Gamma)=e_{L}^{-1}$ for this ideal $L$.

Definition 8. A rigid analytic function $f$ on $\Omega$ is called a modular form of weight $n$ for $\Gamma$ provided that

(1) $f(\gamma z)=\left(c_{\gamma} z+d_{\gamma}\right)^{n} f(z)$ for $\gamma \in \Gamma$.

(2) $f$ is holomorphic at the cusps of $\Gamma$. When the cusp in question is the point $\infty$, this means that $f$ can be expanded as a series

$$
f=\sum_{i \geq 0} a_{i} t(\infty, \Gamma)^{i}
$$

with a nonzero radius of convergence. In general, we require that if $\mu(\infty)=p$ then we have a convergent series

$$
\left(c_{\mu} z+d_{\mu}\right)^{-n} f(\mu z)=\sum_{i \geq 0} a_{i} t(p, \Gamma)^{i} .
$$

A modular form is called a cusp form if it vanishes at the cusps; this means that, in the expansions as in (2) above, we have $a_{0}=0$.

We let $M_{n}(\Gamma)$ denote the space of modular forms of weight $n$ for $\Gamma$ and $S_{n}(\Gamma)$ the space of cusp forms of weight $n$.

Residues. Suppose $e$ is an oriented edge of $\mathscr{T}$. As we discussed earlier, the set of points $V(e)$ in $\Omega$ that reduce to $e$ forms an "annulus"-that is, a rigid domain that is analytically isomorphic to the standard annulus

$$
V=\left\{z \in \Omega: q^{\delta}>|z|>1\right\} .
$$

If $f d z$ is a rigid differential on $V(e)$, we can choose an isomorphism $v$ between $V(e)$ and $V$ that represents the orientation on $e$ (see Definition 1) and then expand

$$
f=\sum_{n \in \mathbb{Z}} a_{n} v^{n} d v .
$$

Definition 9. The residue $\operatorname{Res}_{e} f d z$ is defined to be the coefficient $a_{-1}$ in the expansion of $f$ in equation (2).

The residue map gives a way to construct harmonic cocycles out of modular forms.

Definition 10. Suppose $f$ is a modular form of weight $n$ for an arithmetic subgroup $\Gamma \subset \mathrm{GL}_{2}(k)$. Define a harmonic cocycle $\operatorname{Res}(f)$ of weight $n$ by

$$
\operatorname{Res}(f)(e)\left(X^{i} Y^{n-2-i}\right)=\operatorname{Res}_{e} z^{i} f(z) d z .
$$


$\operatorname{Res}(f)$ is harmonic by the rigid analytic residue theorem, and $\Gamma$-equivariant because of the modular property of $f$. (See Definition 2.)

The first main result of this paper is the following integral formula for cusp forms.

Theorem 11. Let $c$ be a harmonic cocycle of weight $n \geq 2$ for arithmetic $\Gamma \subset$ $\mathrm{GL}_{2}(k)$, and let $\mu$ be the associated measure. Define $F$ by the integral

$$
F(z)=\int_{\mathbb{P}_{k}^{1}} \frac{1}{z-x} d \mu .
$$

Then $F$ is a rigid analytic cusp form for $\Gamma$ of weight $n$ and $\operatorname{Res}(F)=c$.

Proof. The fact that $F$ is a modular form and that $\operatorname{Res}(F)=c$ follows precisely as in the proof of [T, Theorem 3]. To see the modular property, start with the relation

$$
\frac{1}{\gamma z-\gamma x}=\operatorname{det}(\gamma)^{-1} \frac{(c z+d)(c x+d)}{z-x} .
$$

From this, one computes

$$
\begin{aligned}
F(\gamma z) & =\int \frac{1}{\gamma z-x} d \mu(x) \\
& =\int \frac{1}{\gamma z-\gamma x} d \mu(\gamma x) \\
& =\int \frac{(c z+d)(c x+d)^{k-1}}{z-x} d \mu(x) \\
& =\int\left(\frac{(c z+d)^{k}}{z-x}+\sum_{i=0}^{n-2} a_{i}(z) x^{i}\right) d \mu(x) \\
& =(c z+d)^{k} F(z) .
\end{aligned}
$$

Here we have used the transformation property of the measure $\mu$ and the fact that the integral of a polynomial of degree $n-2$ in $x$ is zero; both of these properties are given in Lemma 5.

To verify that $F$ has the desired residues, see Corollary 14, where this fact (and indeed even more) is obtained.

The only new result here is the assertion that $F$ is in fact a cusp form. In Lemma 13, we will prove that $F$ vanishes at $\infty$. This in fact suffices to show that $F$ is a cusp form. To see this, suppose $p$ is another cusp and $\gamma \in \mathrm{GL}_{2}(k)$ is such that $\gamma p=\infty$. Then it suffices to prove that $F^{*}=\left(c_{\gamma} z+d_{\gamma}\right)^{-n} F(\gamma z)$ vanishes at $\infty$. However, a computation using the properties of the integral shows that

$$
\left(c_{\gamma} z+d_{\gamma}\right)^{-n} F(\gamma z)=\int_{\mathbb{P}_{k}^{1}} \frac{\left(c_{\gamma} z+d_{\gamma}\right)^{-n}}{\gamma z-x} d \mu=\int_{\mathbb{P}_{k}^{1}} \frac{1}{z-x} d \mu^{*},
$$

where

$$
\mu^{*}=\operatorname{det}(\gamma)^{-1}(c x+d)^{2-n} \gamma^{*} \mu .
$$


A further check shows that if $\mu$ is derived from the harmonic cocycle $c$, then $\mu^{*}$ is derived from $\gamma c$. Consequently, the proof that $F$ vanishes at infinity, applied to the measure $\mu^{*}$, implies that $F^{*}$ vanishes at infinity as well.

We must introduce some notation before proving that the $F$ defined in Theorem 11 is in fact a cusp form. Recall that the "imaginary absolute value" is defined as

$$
|z|_{i}=\inf _{a \in \hat{k}}|z-a| .
$$

Define the domains $\Omega_{r} \subset \Omega$ by setting

$$
\Omega_{r}=\left\{z \in \Omega:|z|_{i} \geq q^{\delta r}\right\} \quad r \in \mathbb{Z} .
$$

Let $\Gamma_{\infty}$ be the unipotent subgroup of $\Gamma$ that fixes $\infty$. Then the sets $\Omega_{r} / \Gamma_{\infty}$, for $r$ sufficiently large, are a nested sequence of punctured balls around the point at infinity in $\Omega / \Gamma$. (See [Gek1, Paragraph 5.6].)

The following technical lemma supplies the estimate necessary in the proof of Theorem 11.

Lemma 12. Let $F$ be defined by an integral as in Theorem 11. Then there is a constant $C_{1}$, independent of $r$, such that

$$
\sup _{z \in \Omega_{r}}\left|z^{n / 2} F(z)\right| \leq C_{1} .
$$

Consequently, $F$ vanishes at infinity.

Proof. Let $B_{r}(\infty)=\left\{z \in K:|z|>q^{\delta r}\right\}$, and, for an integer $s>r$, define

$$
\Omega_{r, s}=\Omega_{r}-B_{s}(\infty) \text {. }
$$

Then $\Omega_{r, s}$ is the complement in $\mathbb{P}_{K}^{1}$ of $B_{s}(\infty)$ and finitely many other open balls $B_{r}(a)$ of radius $q^{\delta r}$ and with centers $a \in Q=\left\{a_{1}, \ldots, a_{m}\right\}$, all of which are $k$-rational. Let $U_{r}(a)=B_{r}(a) \cap \mathbb{P}_{\hat{k}}^{1}$ for $a \in Q \cup\{\infty\}$. Notice that the radius of $U_{r}(a)$ is $q^{\delta(r-1)}$ if $a$ is finite and $q^{\delta(-r-1)}$ if $a$ is $\infty$. For $z \in \Omega_{r, s}$ we may write

$$
F(z)=F_{s, \infty}(z)+\sum_{a \in Q} F_{r, a}(z),
$$

where for $a \in Q \cup\{\infty\}$,

$$
F_{r, a}(z)=\int_{U_{r}(a)} \frac{1}{z-x} d \mu .
$$

On $U_{s}(\infty)$ we may expand the integral for $F_{s, \infty}$ as follows:

$$
F_{s, \infty}=-\sum_{j=0}^{\infty}\left(\int_{U_{s}(\infty)} x^{(-j-1)} d \mu\right) z^{j}
$$


Applying the estimate from Proposition 7(2), we see that

$$
\begin{aligned}
\sup _{\Omega_{r, s}}\left|F_{s, \infty}\right| & \leq C \sup _{j} q^{\delta(-s-1)(j+1+(n-2) / 2)} q^{\delta s j} \\
& \leq C q^{-\delta(s+1)(n / 2)} \\
& \leq C q^{-\delta(n / 2)} \sup _{z \in \Omega_{r, s}}|z|^{-n / 2} .
\end{aligned}
$$

Working now with $F_{r, a}$ for $a \in Q$, we obtain

$$
F_{r, a}=\sum_{j=0}^{\infty} \frac{\int_{U_{r}(a)}(x-a)^{j} d \mu}{(z-a)^{j+1}} .
$$

Again applying Proposition 7(3), we obtain

$$
\begin{aligned}
\sum_{\Omega_{r, s}}\left|F_{r, a}\right| & \leq C \sum_{j} q^{\delta(r-1)(j-(n-2) / 2)} q^{-r \delta(j+1)} \\
& \leq C q^{\delta((n-2) / 2-r(n / 2))} \\
& \leq C q^{\delta(n-2) / 2} \sup _{z \in \Omega_{r, s}}|z|^{-n / 2}
\end{aligned}
$$

Combining these, we see that there is a constant $C_{2}$ such that $\left|z^{n / 2} F(z)\right|$ is bounded on $\Omega_{r, s}$ independently of $r$ and $s$. If we let $s \rightarrow \infty$, we obtain our estimate.

Remark. In the notation of the proof of this lemma, we may define

$$
\int_{\hat{k}} f(x) d \mu(x)=\lim _{r \rightarrow \infty} \int_{\mathbb{P}_{\hat{k}}^{1}-U_{r}(\infty)} f(x) d \mu(x) .
$$

As we saw in the proof of the lemma, the integral

$$
F_{r, \infty}(z)=\int_{U_{r}(\infty)} \frac{1}{z-x} d \mu(x)
$$

goes to zero uniformly as $r$ goes to infinity. Consequently, the Poisson integral may be computed by integrating over $\hat{k}$, rather than all of $\mathbb{P}_{\hat{k}}^{1}$.

The following corollary will be crucial in the derivation of our formula for Fourier coefficients.

Corollary 13. Let $f$ be a cusp form obtained from a measure $\mu$ as in Theorem 11. Let $e$ be an edge of $\mathscr{T}$. Suppose that $\infty \notin U(e)$, and let a be a center for $U(e)$. Then for all integers $m$, we have

$$
\operatorname{Res}_{e}(z-a)^{m} f d z=\int_{U(e)}(x-a)^{m} d \mu(x) .
$$

Proof. We have the expansion

$$
f(z)=\int_{U(e)} \frac{1}{z-x} d \mu+\int_{U(-e)} \frac{1}{z-x} d \mu,
$$


which converges on the annulus $V(e) \subset \Omega$ corresponding to $e$. On $U(e)$ we have $|x-a| \leq \rho$, while on $U(-e)$ we have $|x-a| \geq q^{\delta} \rho$. In the middle, on $V(e)$, we have $\rho<|z-a|<q^{\delta} \rho$. Expanding the integrals in our formula for $f$, we obtain

$$
\int_{U(e)} \frac{1}{z-x} d \mu=\sum_{i \geq 0} \frac{\int_{U(e)}(x-a)^{i} d \mu}{(z-a)^{i+1}}
$$

and

$$
\int_{U(-e)} \frac{1}{z-x} d \mu=-\sum_{i \geq 0}(z-a)^{i} \int_{U(-e)}(x-a)^{(-i-1)} d \mu .
$$

From this we read off the desired residues immediately.

\section{MEASURES AND MODULAR FORMS}

In this section we examine in detail the measures and modular forms coming from congruence subgroups of $\mathrm{GL}_{2}(k)$. We will show that the kernel of the Res map on such forms is precisely the subspace of Eisenstein series, and that the integral transform in Theorem 11 establishes an isomorphism between $S_{n}(\Gamma)$ and $C_{\text {har }}(\Gamma, n)$. We will rely on information about the Euler characteristic of $\Gamma$, as described in [Se, Chapter II.2.9].

As a first step, we point out that the Eisenstein series have no residues.

Lemma 14. Let $E_{n}$ be an Eisenstein series for $\operatorname{GL}(Y, I)$ of weight $n$. (See [Gek2, p. 47].) Then $\operatorname{Res}\left(E_{n}\right)=0$.

Proof. This is clear from the definition of $E_{n}$. The Eisenstein series are sums of terms of the form $1 /(a z+b)^{n}$, and the differential form $z^{i} d z /(a z+b)^{n}$ clearly has no residues on $\mathscr{T}$ as long as $0 \leq i \leq n-2$.

Let $\bar{M}_{I}$ be the complete algebraic curve obtained by compactifying the quotient $\Omega / \Gamma(I)$, and let $g(I)$ be its genus. Let $h(I)$ be the number of cusps for $\Gamma(I)$, and let $\chi(I)$ denote the Euler-Poincaré characteristic of $\Gamma(I)$ in the sense of [Se, II.2.9]. These invariants are related in the following way (see for example [Gek2, VII.5.11]):

$$
1-g(I)=h(I)+\chi(I) .
$$

We apply this result to obtain the following formula for the number of cusp forms.

Lemma 15. Let $n \geq 2$ be an integer. Then

$$
\operatorname{dim} S_{n}(\Gamma(I))=(1-n) \chi(I) .
$$

Proof. There is an invertible sheaf $\omega$ of degree $g(I)-1+h(I)$ on $\bar{M}_{I}$ such that

$$
S_{n}(\Gamma(I))=\mathrm{H}^{0}\left(\omega \otimes(\text { cusps })^{-1}\right)
$$


(see [Go2, Theorem 1.79 and Corollary 1.81; note the sign error]). By RiemannRoch, therefore, we have

$$
\operatorname{dim} S_{n}(\Gamma(I))=n(g(I)-1+h(I))+1-g(I)-h(I)=(1-n) \chi(I) .
$$

Theorem 16. Let $\Gamma$ be an arithmetic subgroup of $\mathrm{GL}_{2}(k)$, and suppose $n \geq 2$ is an integer. Then the maps

$$
\text { Res: } S_{n}(\Gamma) \rightarrow C_{\text {har }}(\Gamma, n)
$$

and

$$
\imath: C_{\mathrm{har}}(\Gamma, n) \rightarrow S_{n}(\Gamma), \quad c \mapsto \int_{\mathbb{P}_{k}^{1}} \frac{1}{z-x} d \mu_{c}(x)
$$

are mutually inverse isomorphisms.

Proof. It suffices to assume that $\Gamma=\Gamma(I)$ for an ideal $I$, since every $\Gamma$ contains a $\Gamma(I)$ as a subgroup of finite index, and therefore every $f$ for $\Gamma$ is in fact a cusp form for some $\Gamma(I)$. We know that $l$ is injective, and that Res is a left inverse. By Lemma 15 , it suffices to prove that

$$
\operatorname{dim} C_{\text {har }}(\Gamma(I), n) \geq(1-n) \chi(I) .
$$

We will prove this estimate below.

Corollary 17. Let $f$ be a modular form of weight $n \geq 2$ for $\Gamma$. Then $\operatorname{Res}(f)=$ 0 if and only if $f$ lies in the space of Eisenstein series. The intersection of the space of Eisenstein series with the space of cusp forms is zero.

Apparently, the statement we give in this corollary was not known in this generality before.

In order to complete the proof the Theorem 16, we must introduce some terminology. Following Serre [Se, p. 132], we say that a vertex or edge of $\mathscr{T}$ is $\Gamma(I)$-stable if its stabilizer group is trivial, and $\Gamma(I)$-unstable otherwise. Let $\mathscr{T}_{\infty}$ be the subgraph of $\mathscr{T}$ consisting of unstable edges and vertices, and define

$$
S_{0}=\operatorname{Vert}(\mathscr{T})-\operatorname{Vert}\left(\mathscr{T}_{\infty}\right) \text { and } S_{1}=\operatorname{Edge}(\mathscr{T})-\operatorname{Edge}\left(\mathscr{T}_{\infty}\right) \text {. }
$$

Theorem 18. Let $l_{0}=\operatorname{Card}\left(S_{0} / \Gamma(I)\right)$ and $l_{1}=\operatorname{Card}\left(S_{1} / \Gamma(I)\right)$. Then $l_{0}$ and $l_{1}$ are finite and $l_{0}-l_{1}=\chi(I)$.

Proof. This is [Se, Chapter II, Theorem 13'].

Let $C_{\mathrm{har}}^{\text {stable }}(\Gamma(I), n)$ be the space of $\Gamma(I)$-equivariant, $V(1-n)$-valued functions on the stable edges of $\mathscr{T}$, which are harmonic relative to the stable vertices. Such a function is determined by specifying $n-1$ elements of $k$ for each $\Gamma(I)$-class of stable edges; each stable vertex imposes at most $n-1$ linear conditions on the values of $f$. We have proved the following lemma.

Lemma 19. $\operatorname{dim} C_{\text {har }}^{\text {stable }}(\Gamma(I), n) \geq(n-1)\left(l_{1}-l_{0}\right)=(1-n) \chi(I)$.

To prove the inequality (3), and thereby complete the proof of Theorem 16 , we must construct a harmonic cocycle on all of $\mathscr{T}$, given its values on the 


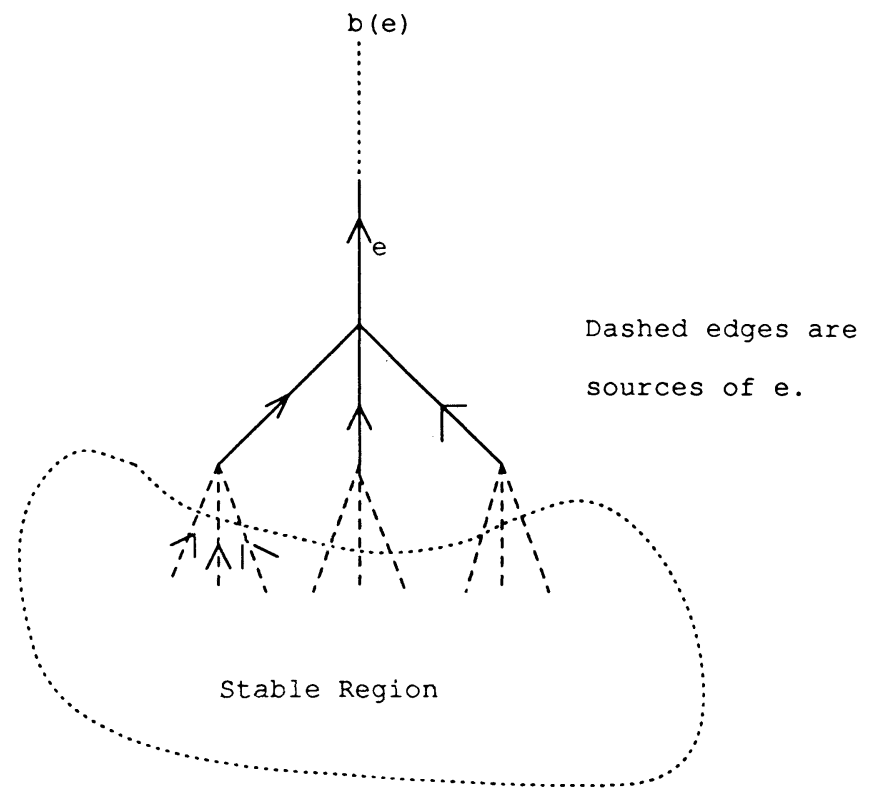

FIGURE 1

stable edges. As explained in [Se, Chapter II, Lemma 13], the stabilizer of an unstable edge $e$ fixes a unique rational end $b(e)$ of the tree $\mathscr{T}$; the stabilizer of an unstable vertex $v$ also fixes a rational end $b(v)$. Let us say that an edge $w$ of $\mathscr{T}$ is a source of an unstable edge $e$ if exactly one boundary vertex $v$ of $w$ is unstable and $b(v)=b(w)$. The source edges of $e$ should be oriented in the same way as $e$, relative to $b(e)$; that is, if $e$ points towards $b(e)$, then so should the sources of $e$. If $e$ is stable, then we will say that $e$ is its own source. The reader may find Figure 1 helpful.

Lemma 20. Let $\operatorname{Src}(e)$ be the sources of $e$. Then

(1) $\operatorname{Src}(\gamma e)=\gamma \operatorname{Src}(e)$.

(2) $\operatorname{Src}(e)$ is finite.

(3) Suppose that $e$ is unstable. Let $v$ be the vertex of $e$ farthest from $b(e)$, and let $e_{1}, \ldots, e_{q^{\delta}}$ be the $q^{\delta}$ edges other than $e$ meeting $v$. Then

$$
\operatorname{Src}(e)=\coprod_{i} \operatorname{Src}\left(e_{i}\right) .
$$

Proof. The first claim is clear. For the second, let $e$ be an unstable edge of $\mathscr{T}$, and let $X(e)$ be the connected component of $\mathscr{T}_{\infty}$ containing $e$. Then, by [Se, $o p$. cit.], $b(e)$ is the only end of $X(e)$. Consequently, every path leaving $e$ and heading away from $b(e)$ reaches a stable edge after travelling a finite distance. This proves the second claim. To verify the third claim, suppose that $w$ is a source of both $e_{i}$ and $e_{j}$. Then the path to $b(e)$ from $w$ passes through both of $e_{i}$ and $e_{j}$, and therefore $e_{i}=e_{j}$. 
Now suppose $f \in C_{\text {har }}^{\text {stable }}(\Gamma(I), n)$. We will show how to extend $f$ to an element of $C_{\text {har }}(\Gamma(I), n)$. Let $\bar{f}$ be defined on the edges of $\mathscr{T}$ by the formula

$$
\bar{f}(e)=\sum_{w \in \operatorname{Src}(e)} f(w)
$$

By Lemma $20, \bar{f}$ is well defined and $\Gamma(I)$-invariant. To see that it is harmonic, suppose that $v$ is an unstable vertex. Let $e$ be the unique edge meeting $v$ that is closest to $b(v)$, oriented towards $b(v)$. Let $e_{1}, \ldots, e_{q^{\delta}}$ be the other $q^{\delta}$ edges meeting $v$, oriented toward $v$ (and therefore toward $b(v)$.) Then, from Lemma 20(3), we see that

$$
\bar{f}(e)=\sum \bar{f}\left(e_{i}\right) .
$$

Consequently, $\bar{f}$ is harmonic. This completes the proof of the inequality (3).

A homological interpretation. The results of this section can be interpreted in terms of the relative homology of $\Gamma$ (see [Se, II.2.9]), when $\Gamma$ is without torsion of order prime to $p$ (this is true, for example, when $\Gamma=\Gamma(I)$ and $I \neq A$ ). Let St (the "Steinberg Module" for $\Gamma$; see [Se, loc. cit.]) be the kernel of the boundary map from the module $L_{1}=\mathbb{Z}\left[S_{1}\right]$ of stable 1-chains to the module $L_{0}=\mathbb{Z}\left[S_{0}\right]$ of stable 0 -chains. We have the exact sequence

$$
0 \rightarrow \mathrm{St} \rightarrow L_{1} \rightarrow L_{0} \rightarrow 0 \text {. }
$$

When $n \geq 2$, there is a map

$$
\phi: C_{\text {har }}(\Gamma, n) \rightarrow L_{1} \otimes_{\Gamma} V(1-n)
$$

defined by

$$
\phi(f)=\sum_{e \in S_{1} / \Gamma} e \otimes f(e) .
$$

This map is well defined (i.e., it does not depend on the choice of representatives for the edges in $S_{1} / \Gamma$ ) and injective, and the fact that $f$ is harmonic implies that $\phi(f)$ actually belongs to $\operatorname{St} \otimes_{\Gamma} V(1-n)$. Furthermore, by Lemma 20, we see that $\phi$ is surjective. However, the group $\mathrm{St} \otimes_{\Gamma} V(1-n)$ is precisely the relative homology group $\mathrm{H}_{1}\left(\Gamma,\left\{\Gamma_{\sigma}\right\}, V(1-n)\right)$ where the $\Gamma_{\sigma}$ are the stabilizers of a system of representatives for the cusps of $\Gamma$. Consequently, we have established the following result.

Proposition 21. The map $\phi \circ$ Res: $S_{n}(\Gamma) \rightarrow H_{1}\left(\Gamma,\left\{\Gamma_{\sigma}\right\}, V(1-n)\right)$ is an isomorphism.

This result provides generalization to the arithmetic subgroups of $\mathrm{GL}_{2}(k)$, of work of Mitchell, Priddy, and Kuhn (see [KM, MP]) on the occurrence of the Steinberg module for the finite group $\mathrm{GL}_{n}\left(\mathbb{F}_{q}\right)$ in the symmetric algebra over $\mathbb{F}_{q}$.

To illustrate this, let $\bar{V}(n)$ be the symmetric forms of degree $n-1$ over the finite field $\mathbb{F}_{q}$, and let $\overline{\mathrm{St}}$ denote the Steinberg module for $\mathrm{GL}_{2}\left(\mathbb{F}_{q}\right)$. This 


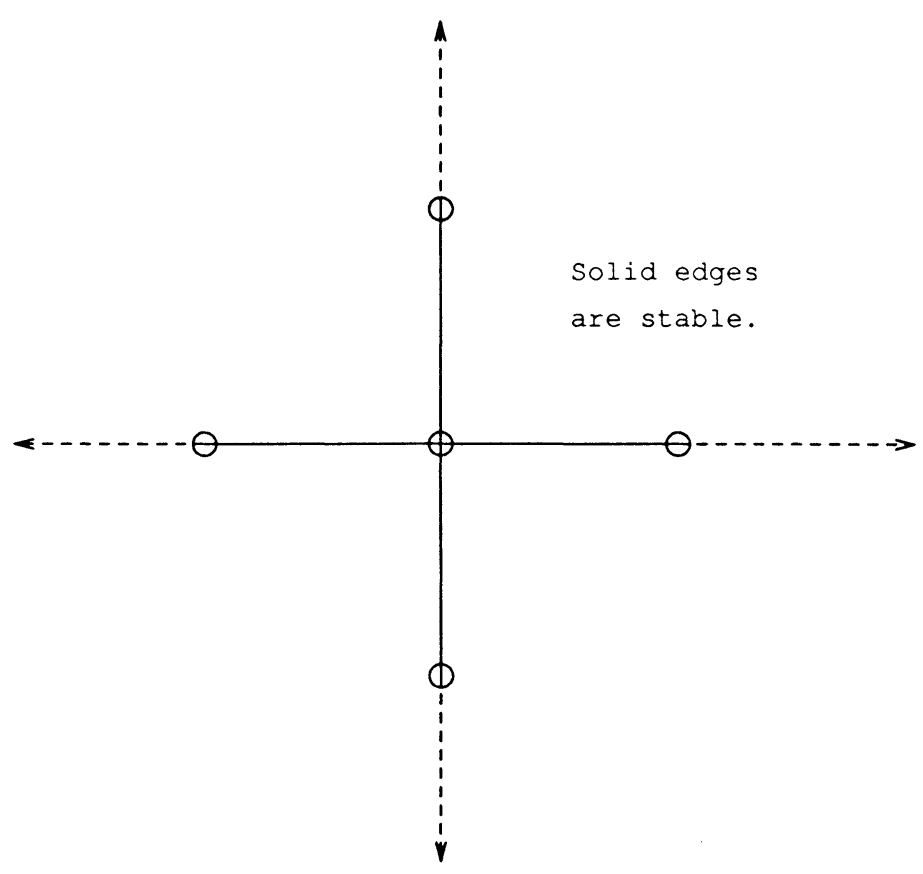

Figure 2. $\mathscr{T} / \Gamma(I)$ when $I=T \mathbb{F}_{3}[T]$.

module is the divisors of degree zero supported on the rational points of $\mathbb{P}_{\mathbb{F}_{q}}^{1}$, with coefficients in $\mathbb{F}_{q}$. Re-indexing Kuhn and Mitchell's series slightly, we can summarize their results as follows. The series

$$
P(t)=\sum a_{n} t^{n}
$$

where

$$
\begin{aligned}
a_{n} & =\operatorname{dim}_{\mathbb{F}_{q}} \operatorname{Hom}_{\mathrm{GL}_{2}\left(\mathbb{F}_{q}\right)}(\overline{\mathrm{St}}, \bar{V}(n-1)) \\
& =\operatorname{dim}_{\mathbb{F}_{q}} \overline{\mathrm{St}} \otimes_{\mathrm{GL}_{2}\left(\mathbb{F}_{q}\right)} \bar{V}(1-n)
\end{aligned}
$$

is the rational function

$$
P(t)=\frac{t^{q+1}}{\left(1-t^{q-1}\right)\left(1-t^{q^{2}-1}\right)} .
$$

We will show how this result can be deduced from Theorem 16. Let $k=$ $\mathbb{F}_{q}(T), A=\mathbb{F}_{q}[T]$, and $\Gamma=\Gamma(I)$ where $I=T A$. The quotient graph $\mathscr{T} / \Gamma$ for $q=3$ is shown in Figure 2; observe that $\Gamma$ has $q+1$ cusps corresponding in a natural way to the points of $\mathbb{P}_{\mathbb{F}_{q}}^{1}$. The group $\mathrm{GL}_{2}\left(\mathbb{F}_{q}\right)$ acts on $C_{\text {har }}(\Gamma, n)$, and we can see this action as the action of $\mathrm{GL}_{2}\left(\mathbb{F}_{q}\right)$ on the $q+1$ classes of $\Gamma$-stable edges in $\mathscr{T}$. Using this, it is not hard to verify that

$$
C_{\text {har }}(\Gamma, n)^{\mathrm{GL}_{2}\left(\mathbb{F}_{q}\right)}=\hat{k} \otimes_{\mathbb{F}_{q}} \overline{\mathrm{St}} \otimes_{\mathrm{GL}_{2}\left(\mathbb{F}_{q}\right)} \bar{V}(1-n) .
$$


Therefore

$$
a_{n}=\operatorname{dim}_{\hat{k}} C_{\mathrm{har}}(\Gamma, n)^{\mathrm{GL}_{2}\left(\mathbb{F}_{q}\right)} .
$$

Now let $S_{n}\left(\mathrm{SL}_{2}(A)\right)^{(1)}$ be the subspace of $S_{n}\left(\mathrm{SL}_{2}(A)\right)$ consisting of modular forms that satisfy

$$
f(a z)=a^{-1} f(z)
$$

for elements $a \in A^{\times}$. (Gekeler calls such forms "modular forms for $\mathrm{GL}_{2}(A)$ of type one," see [Gek1, 5.7].) It is not hard to check that $f \in S_{n}(\Gamma)$ actually belongs to $S_{n}\left(\mathrm{SL}_{2}(A)\right)^{(1)}$ if and only if $\operatorname{Res}(f)$ is $\mathrm{GL}_{2}\left(\mathbb{F}_{q}\right)$ invariant. As a result,

$$
a_{n}=\operatorname{dim} S_{n}\left(\operatorname{SL}_{2}(A)\right)^{(1)} \text {. }
$$

This enables us to interpret the series $P(t)$ in terms of modular forms. More precisely, let $N=\bigoplus_{n>2} S_{n}\left(\mathrm{SL}_{2}(A)\right)^{(1)}$ be the graded module of modular forms of type one over the ring $M=\bigoplus M_{n}\left(\mathrm{GL}_{2}(A)\right)$ of modular forms for $\mathrm{GL}_{2}(A)$. We know that $M$ is generated by an Eisenstein series of weight $q-1$ and by a cusp form of weight $q^{2}-1$ (see [Gol]) and that $N$ is generated over $M$ by the Poincare series $P_{q+1}$ of weight $q+1$ (see [Gek1, 5.13]). Consequently,

$$
P(t)=\sum\left(\operatorname{dim} S_{n}\left(\mathrm{SL}_{2}(A)\right)^{(1)}\right) t^{n}=\frac{t^{q+1}}{\left(1-t^{q-1}\right)\left(1-t^{q^{2}-1}\right)} .
$$

AN INTEGRAL FORMULA FOR THE TAYLOR COEFFICIENTS OF CUSP FORMS

In this section we will apply the theory of measures developed earlier in the paper to derive an integral formula for the Taylor coefficients of a cusp form. We must limit ourselves to a somewhat special situation. Throughout this section, we suppose that $k=\mathbb{F}_{q}(T), A=\mathbb{F}_{q}[T]$, and that $\hat{k}$ is the completion of $k$ relative to the absolute value "at infinity," normalized by $|T|=q$.

Suppose that $f \in M_{n}(\Gamma(I))$ is a modular form, and let

$$
t_{I}(z)=\sum_{a \in I} \frac{1}{z-a} .
$$

The function $t_{I}$ is the logarithmic derivative of $e_{I}$ and functions as a uniformizing parameter "at infinity." The modular form $f$ has an expansion

$$
f(z)=\sum_{i \geq 1} a(i) t_{I}(z)^{i},
$$

which converges on some neighborhood of infinity. The coefficients $a(i)$ are called the Fourier coefficients of $f$ by analogy with the classical theory of modular forms. Some of these coefficients for some modular forms have been calculated "by hand" by Goss [Go2] and Gekeler [Gek1].

We state our formula in terms of the cusp at infinity. Suitable conjugation will move any cusp to infinity, so our formula can be applied, with modification, to any cusp. 
Theorem 22. Let $f \in S_{n}(\Gamma(I))$ be a cusp form of weight $n$. The Fourier coefficients of $f$, as in equation (4), are given by the formula

$$
a(i)=\int_{\hat{k} / I} e_{I}(x)^{i-1} d \mu_{f}(x),
$$

where $\mu_{f}$ is the measure associated to the harmonic cocycle $\operatorname{Res}(f)$.

Before proving Theorem 22, we mush establish some lemmas. The first of these is due to Goss, although we require a slightly more precise version than that given by him.

Lemma 23. Let $B \subset \Omega$ be the region

$$
B=\left\{z \in \Omega:|z|_{i}>q^{\operatorname{deg}(I)-1}\right\} .
$$

The group of I-translations stabilizes $B$, and $t_{I}$ maps $B / I$ isomorphically onto the set $\left\{t \in K-0:|t|<q^{1-\operatorname{deg}(I)}\right\}$.

Proof. If $z \in B$, we have, for all $a \in I$,

$$
|z-a| \geq|z|_{i}>q^{\operatorname{deg}(I)-1}
$$

and therefore $\left|t_{I}\right|<q^{1-\operatorname{deg}(I)}$. On the other hand, suppose $|z|_{i} \leq q^{\operatorname{deg}(I)-1}$. Let $b \in \hat{k}$ be such that $|z-b|=|z|_{i}$. There is a unique $a \in I$ such that $|b-a| \leq q^{\operatorname{deg}(I)-1}$. We therefore have $\left|t_{I}(z)\right| \geq q^{\operatorname{deg}(I)-1}$. Since $t_{I}^{-1}$ is known to be an analytic isomorphism from $K / I$ to $K$, this proves the lemma. (See [Go2, Thereom 1.76].)

We are now in a position to give the proof of Theorem 22.

Proof of Theorem 22. Let $e$ be the oriented edge of $\mathscr{T}$ corresponding to the region $\mathscr{M}=\left\{x \in \hat{k}:|x|<q^{\operatorname{deg}(I)}\right\} . \mathscr{M}$ is a fundamental domain for $I$ in $\hat{k}$. The region $V(e) \subset \Omega$, which reduces to $e$, is the annulus $V(e)=\left\{z: q^{\operatorname{deg}(I)}>\right.$ $\left.|z|>q^{\operatorname{deg}(I)-1}\right\}$. Now $V(e)$ is contained in the set $B$ of the preceding lemma, so the Fourier expansion of $f$ converges on $V(e)$. In addition, the function $t_{I}^{-1}=e_{I}$ is an analytic parameter on $V(e)$. To see this, write

$$
t_{I}(z)=\sum_{a \in I} \frac{1}{z-a} .
$$

Then, since $|z|<q^{\operatorname{deg}(I)}$ on $V(e)$, we know that, for $a \neq 0,|z-a|=|a| \geq$ $q^{\operatorname{deg}(I)}$. Therefore $t_{I}(z)=z^{-1} u(z)$ where $u(z)$ is close to 1 on $V(e)$. Now we observe that if $f=\sum a(i) t_{A}^{i}$ then

$$
a(i)=\operatorname{Res}_{e} e_{I}^{i-1} f d e_{I}=\operatorname{Res}_{e} e_{I}^{i-1} f d z .
$$

(Recall that $e_{I}$ is additive, and so $d e_{I}=d z$.) Expand $e_{I}^{i-1}$ in a convergent series

$$
e_{I}^{i-1}(z)=\sum u_{i, j} z^{j}
$$


Substituting this into our formula for $a_{i}$, and using Corollary 13 , we compute

$$
\begin{aligned}
a(i) & =\operatorname{Res}_{e} e_{I}^{i-1} f d z=\sum_{j} \operatorname{Res}_{e}\left(u_{i, j} z^{j} f d z\right) \\
& =\sum_{j} \int_{U(e)} u_{i, j} x^{j} d \mu_{f}(x)=\int_{U(e)} \sum_{j} u_{i, j} x^{j} d \mu_{f}(x) \\
& =\int_{U(e)} e_{I}^{i-1}(x) d \mu_{f}(x)=\int_{\mathscr{M}} e_{I}^{i-1}(x) d \mu_{f}(x) . \\
& =\int_{\hat{k} / I} e_{I}^{i-1} d \mu_{f}(x) .
\end{aligned}
$$

The interchange of summation and integration is justified by Proposition 7(3), using the convergence of the expansion for $e_{I}^{i-1}$.

Let us make a few remarks about this formula. First, from an arithmetic point of view, $t_{I}$ may not be the most appropriate parameter at infinity. For example, if $I=A$, it is more appropriate to expand $f$ in the parameter $t=t_{A} / \bar{\pi}$, where $\bar{\pi}$ is the "period" of the Carlitz module (see [Gek1, 4.1] for example). Clearly the coefficients of the expansion

$$
f=\sum_{i \geq 1} b_{i} t^{i}
$$

satsify

$$
b_{i}=\bar{\pi}^{i} \int_{\hat{k} / A} e_{A}^{i-1} d \mu_{f}=\bar{\pi} \int_{\hat{k} / A} e(\bar{\pi} x)^{i-1} d \mu_{f},
$$

where $e(x)=1 / t(x)$ is the exponential function for the Carlitz module. This is the formula we gave in the introduction to this paper.

It is also worth noticing what our formula means with regard to a "double" cusp form. A cusp form is called a double cusp form (following [Go2]) if it has a double zero at the cusps. This means that the coefficient $a(1)$ in its Fourier expansion (at each cusp) vanishes. Requiring that $a(1)$ vanish for the cusp at infinity, for example, is the same as requiring that

$$
\int_{\hat{k} / I} d \mu_{f}=\operatorname{Res}_{e}(f)=0,
$$

where $e$ is an edge chosen so that $U(e)$ is a fundamental domain for $k / I$ as in the proof of Theorem 22. It is interesting that the behavior of $f$ at the cusps is determined by its behavior in the interior of $\Omega$.

\section{CONCLUSION}

Our goal in this paper has been to illustrate the power of the "Poisson Kernel" method in the study of rigid analytic modular forms for $\mathrm{GL}_{2}(k)$. Many areas for further research remain. For example, there is a natural map from the space of harmonic cocycles of weight $n$ for $\Gamma$ into the cohomology group $H^{1}(\Gamma, V(1-n))$. In the characteristic zero case, this is an isomorphism. What about for function fields? 
In addition, it would be worthwhile to extend the formula we have given for the Fourier coefficients of a modular form for congruence subgroups of $\mathrm{GL}_{2}\left(\mathbb{F}_{q}[T]\right)$ to more general arithmetic groups.

Another interesting problem is to determine precisely the exact harmonic cocycles attached to certain "known" cusp forms, such as the Poincare series $P_{q+1}$ and the "discriminant function" $\Delta_{q^{2}-1}$ on $\mathrm{SL}_{2}\left(\mathbb{F}_{q}[T]\right)$. The theory we have presented determines these cocycles up to a multiplicative constant in $\hat{k}$.

Finally, the arithmetic implications of this work, and in particular its potential relation to the theory of $L$-functions and other arithmetic objects associated to Drinfeld modular curves, needs to be clarified.

\section{REFERENCES}

[Al] J. L. Alperin, Local representation theory, Cambridge Stud. Adv. Math., vol. 11, Cambridge Univ. Press, Cambridge, New York, 1986.

[D] V. G. Drinfeld, Elliptic modules, Math. USSR. Sb. 94 (1974), 594-627. (Russian); English Transl. Math. USSR-Sb. 23 (1976), 561-592.

[GvdP] L. Gerritzen and M. van der Put, Schottky groups and. Mumford curves, Lecture Notes in Math., vol. 817, Springer, Berlin, Heidelberg, New York, 1980.

[Gek1] E. U. Gekeler, On the coefficients of Drinfeld modular forms, Invent. Math. 93 (1988), 667700.

[Gek2] _ Drinfeld modular curves, Lecture Notes in Math., vol. 1231, Springer, 1986.

[Go1] D. Goss, Modular forms for $\mathbb{F}_{r}[T]$, J. Reine Angew. Math. 317 (1980), 16-39.

[Go2] _ _ ז-adic Eisentstein series for function fields, Compositio Math. 41 (1980), 3-38.

[Go3] _ On a new type of L-function for algebraic curves over a finite field, Pacific J. Math. 105 (1983), 143-181.

[KM] N. Kuhn and S. Mitchell, The multiplicity of the Steinberg representation of $\mathrm{GL}_{2}\left(\mathbb{F}_{q}\right)$ in the symmetric algebra, Proc. Amer. Math. Soc. 96 (1986), 1-6.

[MP] S. Mitchell and S. Priddy, Stable splittings derived from the Steinberg module, Topology 22 (1983), 285-298.

[MTT] B. Mazur, J. Tate, and J. Teitelbaum, On p-adic analogues of the conjecture of Birch and Swinnerton-Dyer, Invent. Math. 84 (1986), 1-48.

[Sch] P. Schneider, Rigid analytic L-transforms, Number Theory ( Proceedings, Noordwijkerhout 1983) Lecture Notes in Math., vol. 1068, Springer, Berlin, Heidelberg, New York, 1983, pp. 216-230.

[Se] J. P. Serre, Trees, Springer, 1980.

[T1] J. Teitelbaum, Values of p-adic L-functions and a p-adic Poisson kernel, Invent. Math. 101 (1990), 395-410.

[T2] _ On Drinfeld's universal formal group over the p-adic upper half plane, Math. Ann. 284 (1989), 647-674.

[W] A. Weil, On the analogue of the modular group in characteristic p, Collected Papers, vol. III, Springer, New York 1979, pp. 201-213.

Department of Mathematics, Statistics, and Computer Science, M/C 249, The UniverSity of Illinois at Chicago, Chicago, IllinoIs 60680

E-mail address: jeremy@math.uic.edu 